\title{
Twisted reduction in large N QCD with two adjoint Wilson fermions
}

\author{
Antonio González-Arroyo ${ }^{a b}$ and Masanori Okawa* \\ a Instituto de Física Teórica UAM/CSIC \\ ${ }^{b}$ Departamento de Física Teórica, C-15 \\ Universidad Autónoma de Madrid, E-28049-Madrid, Spain \\ ${ }^{c}$ Graduate School of Science, Hiroshima University \\ Higashi-Hiroshima, Hiroshima 739-8526, Japan \\ E-mail: antonio.gonzalez-arroyo@uam.es okawa@sci.hiroshima-u.ac.jp
}

The twisted reduced model of large $N$ QCD with two adjoint Wilson fermions is studied numerically using the Hybrid Monte Carlo method. This is the one-site model, whose large $N$ limit (large volume limit) is expected to be conformal or nearly conformal. The symmetric twist boundary condition with flux $k$ is used. $k=0$ corresponds to periodic boundary conditions. It is shown that the quark mass and $N$ dependencies of the model with non-vanishing $k$ differ significantly from those of the $k=0$ model. A preliminary result for the string tension calculated at $N=289$ is presented. The string tension seems to vanish as the physical quark mass decreases to zero in a way consistent with the theory being governed by an infrared fixed point with $\gamma_{*}=0.8 \sim 1.2$.

The 30th International Symposium on Lattice Field Theory

June 24 - 29, 2012

Cairns, Australia

${ }^{*}$ Speaker. 


\section{Introduction}

Recently the $\mathrm{SU}(\mathrm{N})$ lattice gauge theories with two adjoint Wilson fermions have received much attention, since their massless limits are expected to be conformal or nearly conformal[1]. In fact the first two coefficients of the perturbative $\beta$ function expressed in terms of the 't Hooft coupling are given by $b_{0}=\left(11-4 N_{f}\right) / 24 \pi^{2}$ and $b_{1}=\left(17-16 N_{f}\right) / 192 \pi^{4}$, with $N_{f}$ the number of adjoint quark flavors. This would imply the existence of an infrared fixed point if $17 / 16<N_{f}<$ $11 / 4$ irrespective of the value of gauge group $N$. In particular, the $\mathrm{SU}(2)$ theory has been studied by many authors, and now there is a consensus that this theory is conformal and the chiral condensate anomalous dimension at the infrared fixed point is approximately $\gamma_{*} \sim 0.3$ 1].

The purpose of the present talk is to study the large $N$ lattice gauge theory with two adjoint Wilson fermions directly by means of the twisted space-time reduced model[2] 3]. This is a onesite model given by four $\mathrm{SU}(\mathrm{N})$ matrices only. There is a close connection between this model with $N=L^{2}$, with the usual $\mathrm{SU}(\mathrm{N})$ theory on a $L^{4}$ space-time lattice. In the large $L$ limit this model should reproduce the corresponding large $N$ infinite volume theory. It should be noted that the use of the space-time reduced model has a great advantage in the treatment of the dynamical quark effects. It is almost impossible to make a simulation with both a large $\mathrm{SU}(\mathrm{N})$ internal group and large volume $V$, since the number of the degrees of freedom is too large. On the other hand, the number of degrees of freedom is significantly reduced, by a factor $V$, in the space-time reduced model, and the study of the dynamical quark effects is manageable in this theory.

It is widely advocated [4, 5, 6] that the introduction of the dynamical adjoint Wilson fermions is enough to restore the $Z^{4}(N)$ symmetry which is needed for the reduction idea to hold [7], and is broken [8] in the original Eguchi-Kawai model (EK model)[7]. Our results also confirm that, with the inclusion of the dynamical adjoint fermions, the $\mathrm{Z}^{4}(\mathrm{~N})$ symmetry is indeed unbroken for a wide range of values of $N$ and bare quark masses. At the same time, however, we also find that this theory has a very strong $N$ dependence, making it practically useless for extracting the large $\mathrm{N}$ behavior. Our proposal is to apply twisted boundary conditions in the formulation of the space-time reduced model. For the pure gauge theory, we have recently demonstrated that the twisted reduction (the twisted Eguchi Kawai model) works quite well, allowing a determination of the continuum string tension[9 10]. We will show that the model with dynamical adjoint Wilson fermions and twisted boundary conditions has significantly less $N$ dependence than its untwisted counterpart. This has allowed us to present a preliminary calculation of the large $\mathrm{N}$ string tension using $N=289$. We show that the string tension seems to vanish as we decrease the quark mass towards zero, as predicted by the existence of an infrared fixed point. The rate of decrease points towards a large anomalous dimension $\gamma_{*}=0.8 \sim 1.2$ at this point.

\section{Formulation}

We consider $\mathrm{SU}(\mathrm{N})$ gauge group with $N=L^{2}, L$ being a positive integer. Then the action of the model is given by

$$
S=b N \sum_{\mu \neq v=1}^{4} \operatorname{Tr}\left(Z_{\mu \nu} U_{\mu} U_{v} U_{\mu}^{\dagger} U_{v}^{\dagger}\right)+\sum_{j=1}^{N_{f}} \bar{\Psi}_{j} D_{W} \Psi_{j} .
$$

$b$ is the inverse 't Hooft coupling $b=1 / g^{2} N . U_{\mu}$ are four $\mathrm{SU}(\mathrm{N})$ matrices and $\Psi_{j}$ are the fermion fields in the adjoint representation of color $\mathrm{SU}(\mathrm{N})$ for $N_{f}=2$. The twist tensor 


$$
Z_{\mu v}=\exp \left(k \frac{2 \pi i}{L}\right), \quad Z_{v \mu}=Z_{\mu \nu}^{*}, \quad \mu>v
$$

introduces symmetric twisted boundary conditions to the space-time reduced model specified by the flux $k$. The weak coupling analysis demands that $k$ and $L$ are co-prime for the model to work well in that limit [3]. The $k=0$ case corresponds to periodic boundary conditions. The Wilson Dirac matrix is given by

$$
D_{W}=1-\kappa \sum_{\mu=1}^{4}\left[\left(1-\gamma_{\mu}\right) U_{\mu}^{a d j}+\left(1+\gamma_{\mu}\right) U_{\mu}^{a d j \dagger}\right] .
$$

$U_{\mu}^{a d j}$ are the link variables acting on the adjoint fermions in the color $(N, \bar{N})$ representation as $U_{\mu}^{a d j} \Psi_{j}=U_{\mu} \Psi_{j} U_{\mu}^{\dagger}$.

We have studied the previous model by means of the Hybrid Monte Carlo method. The simulation has been done on Hitachi SR16000 super computer with one node having 32-cores IBM power7 and peak speed of 980 GFlops/sec. Thanks to the Hitachi system engineers, our codes are highly optimized for SR 16000 and the sustained seed is 300-600 GFlops/sec depending on the values of $N$ and $\kappa$.

To study the $N$ dependence of the model precisely, we have performed simulations at $N=25$, $49,81,121,169,225,289$, which correspond to all odd integer values of $L$ from 5 to 17 . It is known that part of the finite $N$ effects for our twisted model correspond to the finite volume effects in the usual lattice theory on an $L^{4}$ lattice [2]: Hence, we based our results on Wilson loops $W(R, T)$ up to $R=T=(L-1) / 2$. For $N=289$ this corresponds to $R=T=8$.

\section{3. $\kappa$ and $N$ dependence}

In fig. 1 we show the $\kappa$ dependence of the average of plaquette $E=<Z_{\mu \nu} U_{\mu} U_{v} U_{\mu}^{\dagger} U_{v}^{\dagger}>$ at $b=0.35$. For periodic boundary conditions $k=0$, we show the results for $N=25,49$ and 81 . As $\kappa$

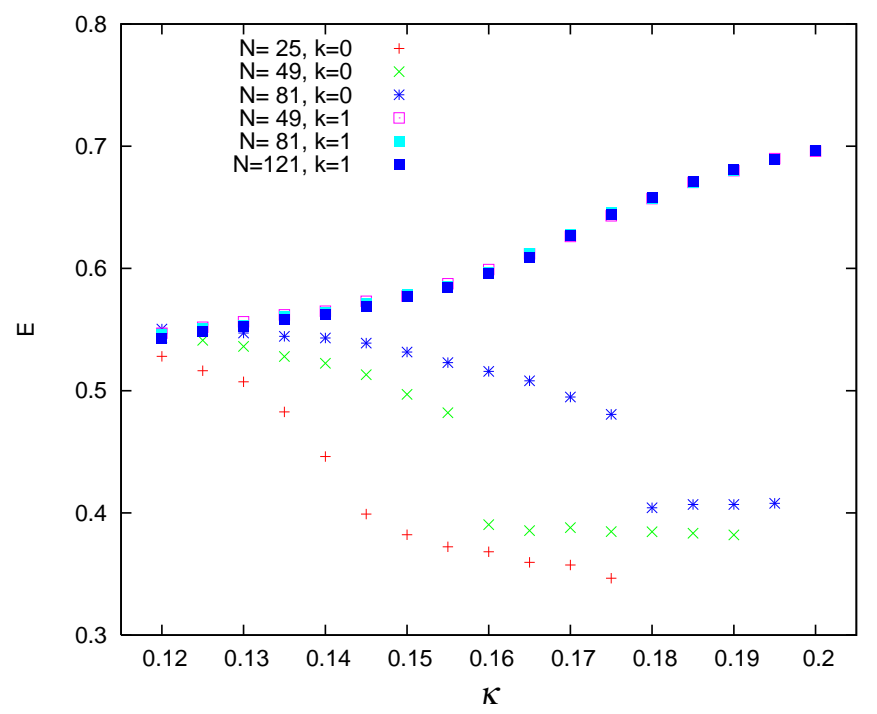

Figure 1: $\kappa$ dependence of $E$ for various $N$ at $b=0.35$ and $k=0$ and 1 . 


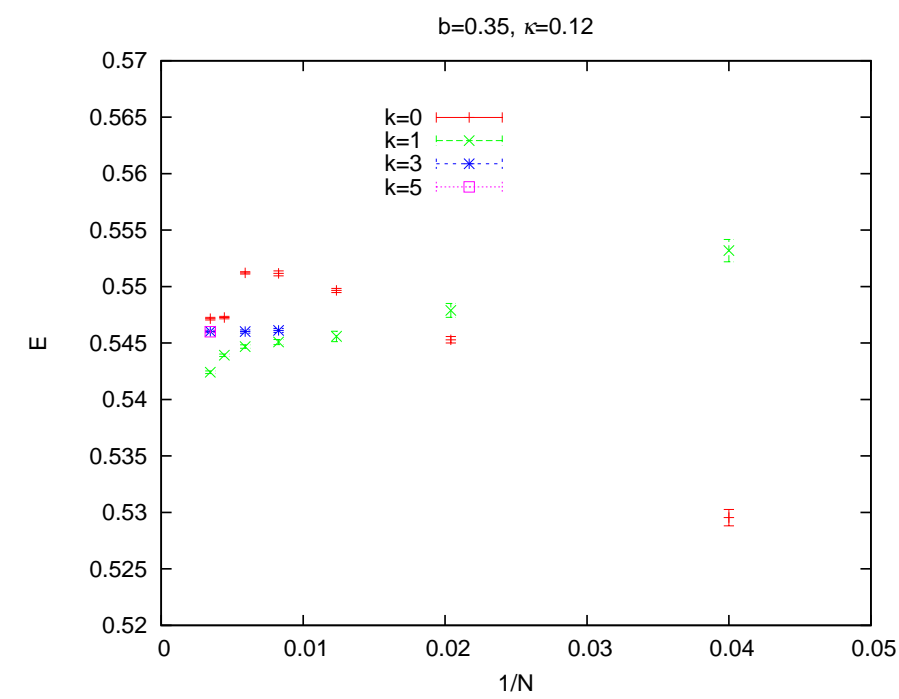

Figure 2: $N$ dependence of $E$ for various $k$ at $b=0.35$ and $\kappa=0.12$.

increases $E$ decreases, which is quite unnatural since as $\kappa$ increases one usually expects to have larger dynamical quark effects which tends to order the system and, thus, to increase the internal energy $E$. Furthermore, there exists an abrupt change in $E$ at intermediate values of $\kappa$. However, the position of the abrupt change depends significantly on the value of $N$. This change is not related to $\mathrm{Z}^{4}(\mathrm{~N})$ symmetry breaking, which was found to remain unbroken for the set of simulation parameters presented in this talk. The large $N$ dependence suggests that it is related to some finite $N$ artifact. The results for twisted boundary conditions and $k=1$ are shown for $N=49,81$ and 121 . In this case $E$ increases as we increase $\kappa$ as expected, and the $N$ dependence looks rather small within the rough scale of the plot.

A more precise study of the $N$ dependence is given in fig. 2, where $E$ is plotted as a function of $1 / N$ for $b=0.35, \kappa=0.12$ and various values of $k$. As we have stated previously, at $\kappa=0.12$ there is no $Z^{4}(\mathrm{~N})$ symmetry breaking. However, the results at finite value of $\kappa$ seem to be strongly affected by the nearby presence of the $\mathrm{Z}^{4}(\mathrm{~N})$ symmetry breaking point at $\kappa=0$, namely the reduced model without fermions. We know that for the pure gauge theory $(\kappa=0)$ the breaking occurs as follows 8 11, 12, 13, 3]:

- $k=0, \mathrm{Z}^{4}(\mathrm{~N})$ symmetry is broken for all $N>0$.

- $k=1, \mathrm{Z}^{4}(\mathrm{~N})$ symmetry is broken for $N>100$.

- $k=3, Z^{4}(\mathrm{~N})$ symmetry is broken for $N>784$.

- $k=5, \mathrm{Z}^{4}(\mathrm{~N})$ symmetry is expected to be broken for $N>2200$.

Now let us look at fig. 2 more carefully. The data with $k=0$ have, again, a large $N$ dependence. For $N \leq 121$, this dependence seems linear in $1 / N$. However, such a linear extrapolation to the large $N$ value of $E$ is quite misleading, since the behavior of $E$ suddenly changes for $N>121$. The data with $k=1$ have less $N$ dependence. However, as we increase beyond $N=121, E$ also starts to decrease. We think this behavior is closely related to the $Z^{4}(N)$ symmetry breaking at $\kappa=0$. If this interpretation is correct, we should expect a similar pattern for $k=3(5)$, with a smooth $N$ dependence 
up to $N \leq 784(N \leq 2200)$. In fact, we observe that the two data points at $N=289$ for $k=3$ and 5 are quite consistent. In fig. 3 and 4 , we display the same type of information but for $(b, \kappa)$ values of $(0.36,0.12)$ and $(0.35,0.14)$ respectively. Details are different, but the overall trends are the same as in Fig. 2.
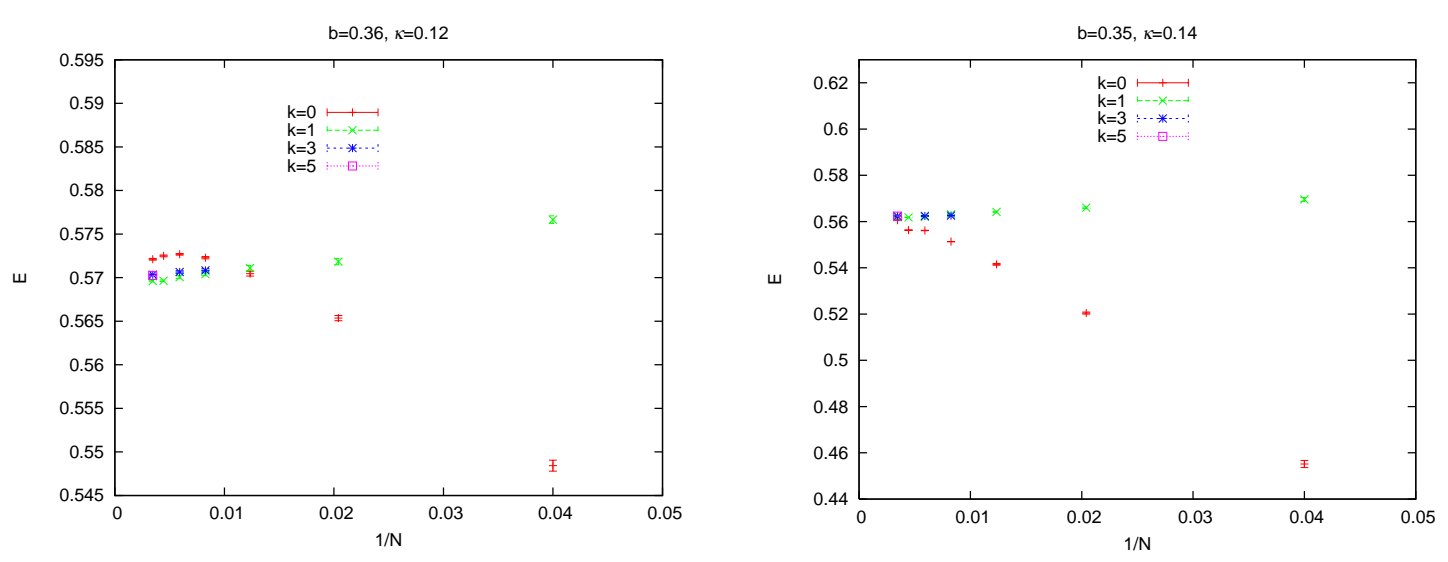

Figure 3: $N$ dependence of $E$ at $b=0.36$ and $\kappa=0.12$. Figure 4: $N$ dependence of $E$ at $b=0.35$ and $\kappa=0.14$.

\section{String tension at $N=289$.}

Based on the results of the previous section, we made an attempt at a determination of the string tension for $N=289$ and $k=5$ and various values of $\kappa$. We used the same procedure as used for the pure gauge reduced model in order to extract the string tension from rectangular $R \approx T$ Wilson loops $W(R, T)$ [10]. Namely, we extract the string tension from Creutz ratios formed out of Wilson loops with smeared links. The Ape smeared links, defined as

$$
U_{\mu}^{\text {smeared }}=\operatorname{Proj}_{N}\left[U_{\mu}+c \sum_{v \neq \mu}\left(Z_{v \mu} U_{v} U_{\mu} U_{v}^{\dagger}+Z_{\mu v} U_{v}^{\dagger} U_{\mu} U_{v}\right)\right],
$$

are used to enhance the signal to noise ratio. $\operatorname{Proj}_{N}$ stands for the operator for projection onto the $\mathrm{SU}(\mathrm{N})$ matrices.

In fig. 5, we show Wilson loops $W(R, T)$ after 5 smearing with smearing parameter $c=0.1$. We observe a clear signal up to loop size 8 , which according to our previous comment, corresponds to slighly less than half the effective linear lattice size $L=17$ for our value of $N=289=17^{2}$.

In fig. 6, we compare Wilson loops calculated with different $k$ values. In this rough scale, Wilson loops with $R=1, W(R=1, T)$, look consistent for all values of $k=0,1,3$ and 5 . On the other hand, the situation is quite different for $R=5$. The results with $k=3$ and 5 coincide with each other, giving what we think are the correct values. It is amazing that the results with $k=0$ are so different from those of $k=3$ and 5 as $T$ increases. The Wilson loops $W(R=5, T)$ with $k=1$ also have smaller values than those with $k=3$ and 5 . We believe that this is also related to the instabilities occurring for $k=1$ at $N>100$ with $\kappa=0$.

In fig. 7, we show with red symbols our preliminary results for the extracted string tension $\sigma$ as a function of $\kappa$ calculated at $N=289, k=5, b=0.35$. The value at $\kappa=0$ is obtained from the Twisted 


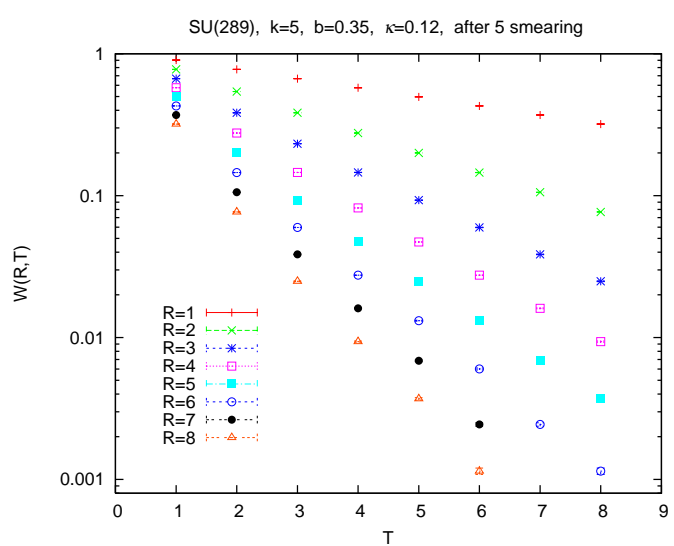

Figure 5: Wilson loop after 5 smearing.

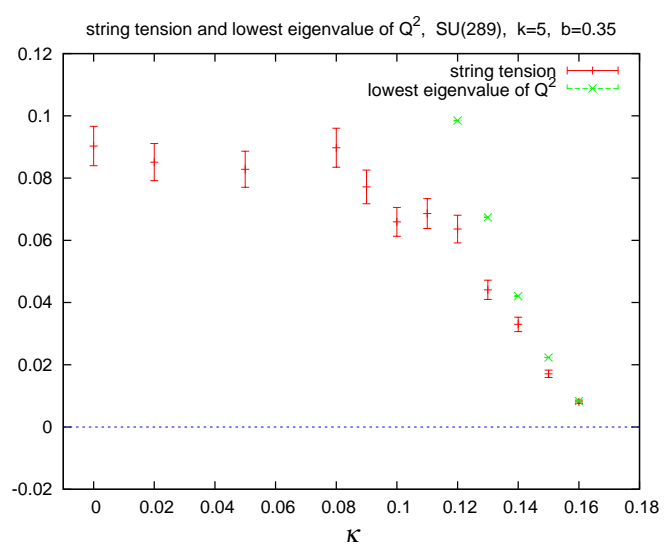

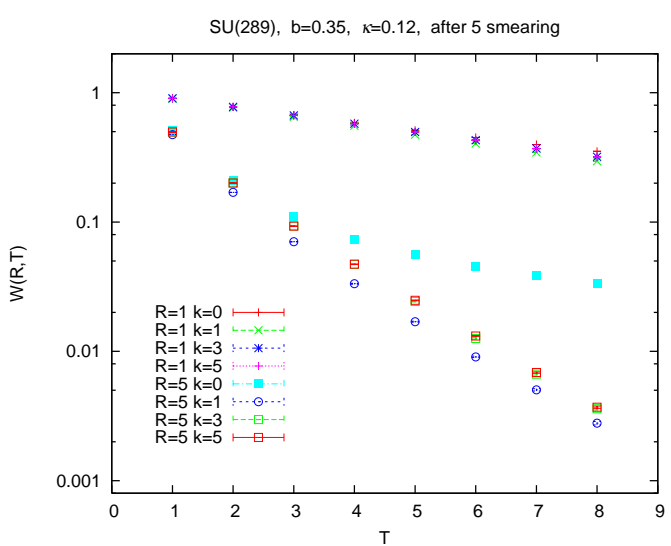

Figure 6: Wilson loop for various values of $k$.

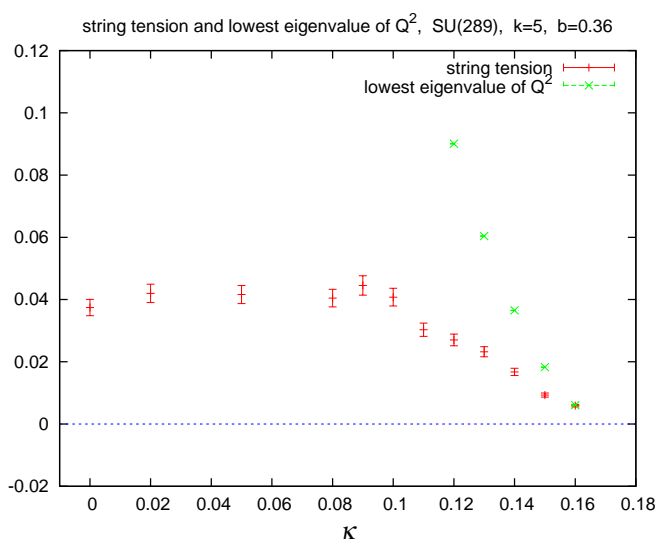

Figure 7: $\sigma$ and lowest eigenvalue of $Q^{2}$ at $b=0.35$. Figure 8: $\sigma$ and lowest eigenvalue of $Q^{2}$ at $b=0.36$.

Eguchi Kawai model without fermions. As we increase $\kappa$, the string tension decreases and seems to vanish around $\kappa=0.17$.

Although at present we have not calculated any fermionic spectrum, it is straightforward to calculate the lowest eigenvalue of the positive square hermitian Wilson-Dirac operator $Q^{2}=\left(\gamma_{5} D_{W}\right)^{2}$, which should be related to quark mass square. In fig. 7, we also plot the lowest eigenvalue of $Q^{2}$ with green symbols. It seems that the string tension and the lowest eigenvalue of $Q^{2}$ vanish at the same point.

If the massless theory is governed by the infrared fixed point at which the theory is conformal, all physical quantities having dimension of mass square should behave as

$$
\sigma \sim\left(\frac{1}{\kappa}-\frac{1}{\kappa_{c}}\right)^{\frac{2}{1+\gamma_{*}}}
$$

with $\gamma_{*}$ the chiral condensate anomalous dimension at the infrared fixed point. We assume that the quark mass is proportional to $\left(1 / \kappa-1 / \kappa_{c}\right)$. Fitting the $b=0.35$ data in the range $\kappa=0.12-0.16$ with a common vanishing point $\kappa=\kappa_{c}$ for the string tension and the lowest eigenvalue of $Q^{2}$, we 
get a rather large estimate for $\gamma_{*}=0.81(8)$. This is considerably larger than the value obtained by other authors for $N=2[1]$. It should be noted, however, that our data of the string tension are quite preliminary. In particular, it is based on small number of configurations of order 100 with a rather low global acceptance ratio in HMC at $\kappa=0.15$ and 0.16 .

In fig. 8, we repeat the analysis for the data at $b=0.36$. Again, requiring that the string tension and the lowest eigenvalue of $Q^{2}$ vanish at the same point, our data for the string tension in the range $\kappa=0.12-0.15$ give $\gamma_{*}=1.17(21)$.

\section{Conclusions}

We have demonstrated that the twisted reduced model of large $N$ QCD with two adjoint Wilson fermions works quite well. The $N$ dependence of the model with twisted boundary conditions $(k \neq 0)$ is significantly smaller than that of the model with periodic boundary condition $(k=0)$. The string tension calculated at $N=289, k=5$, clearly decreases as we increase $\kappa$ and seems to vanish around $\kappa=0.17$ in a way consistent with the expectations for an infrared fixed point with a rather large value for $\gamma_{*}=0.8 \sim 1.2$.

We have started new simulations for small quark mass with reasonable global acceptance ratio. We have also launched the simulation of the single flavor adjoint Wilson fermion using the Rational Hybrid Monte Carlo method. We hope to present new results at the coming lattice conference.

A.G-A is supported from Spanish grants FPA2009-08785, FPA2009-09017, CSD2007-00042, HEPHACOS S2009/ESP-1473, PITN-GA-2009-238353 (ITN STRONGnet) and CPAN CSD200700042. M.O is supported in part by Grants-in-Aid for Scientific Research from the Ministry of Education, Culture, Sports, Science and Technology (No 23540310).

The calculation has been done on Hitachi SR16000-M1 computer at High Energy Accelerator Research Organization (KEK) supported by the Large Scale Simulation Program No.12-01 (FY2011-12).

\section{References}

[1] L. Del Debbio, PoS (Lattice 2010)004.

[2] A. Gonzalez-Arroyo and M. Okawa, Phys. Rev. D27 (1983) 2397.

[3] A. Gonzalez-Arroyo and M. Okawa, JHEP 1007 (2010) 043.

[4] P. Kovtun, M. Unsal, L. G. Yaffe, JHEP 0706 (2007) 019.

[5] T. Azeyanagi, M. Hanada, M. Unsal, R. Yacoby, Phys. Rev. D82 (2010) 125013.

[6] B. Bringoltz, M. Koren, S. R. Sharpe, Phys. Rev. D85 (2012) 094504.

[7] T. Eguchi and H. Kawai, Phys. Rev. Lett. 48 (1982) 1004.

[8] G. Bhanot, U. M. Heller and H. Neuberger, Phys. Lett. B113 (1982) 47.

[9] A. Gonzalez-Arroyo and M. Okawa, arXiv:1206.0049 [hep-th].

[10] A. Gonzalez-Arroyo and M. Okawa, PoS (Lattice 2012) 221

[11] T. Ishikawa and M. Okawa, talk given at the Annual Meeting of the Physical Society of Japan, March 28-31, Sendai, Japan (2003).

[12] M. Teper and H. Vairinhos, Phys. Lett. B652 (2007) 359.

[13] T. Azeyanagi, M. Hanada, T. Hirata and T. Ishikawa, JHEP 0801 (2008) 025. 